\title{
LA FÍSICA, A TRAVÉS DE LA BIOMECÁNICA, COMO EXPLICACIÓN PSÍQUICA DEL ESTRÉS HUMANO
}

\author{
PHYSICS, THROUGH BIOMECHANICS, SUCH AS \\ PSYCHOLOGICAL EXPLANATION OF HUMAN STRESS
}

\section{AUTORES}

Prof. Dr. Horacio Rico Lenza: Departamento de Medicina. Universidad de Alcalá horacio.rico@mixmail.com

\section{CURRICULUM VITAE}

Catedrático de Clínica Médica de la Universidad de Alcalá.

\section{RESUMEN}

El concepto de estrés nace de un principio físico que define que cuando se aplica una fuerza a un objeto este tiende a oponer una resistencia a la misma, lo que los anglosajones denominan stress; la fuerza aplicada y el estrés que se opone a la misma, ocasionan una deformidad en el objeto, que ellos denominan strain.

\section{PALABRAS CLAVE}

Física - Biomecánica - Estrés

\section{ABSTRACT}

The concept of stress arises from a physical principle that defines when a force is applied to an object that tends to oppose a resistance to it, which the Anglo-Saxons 
called stress, the stress applied force that opposes it, cause a deformity in the object, it calls strain.

\section{KEY WORDS}

Physics - Biomechanics - Stress

El concepto de estrés nace de un principio físico que define que cuando se aplica una fuerza a un objeto este tiende a oponer una resistencia a la misma, lo que los anglosajones denominan stress; la fuerza aplicada y el estrés que se opone a la misma, ocasionan una deformidad en el objeto, que ellos denominan strain.

El equilibrio entre la fuerza aplicada y la deformidad inducida en un principio es lineal, la denominada como zona elástica (Fig. 1), si la deformación, y por tanto la fuerza continua, mantienen sus valores bajos. De persistir la fuerza se entra en una zona límite, denominada por los anglosajones como la "yield region", después de la cual, si la fuerza aumenta, se entra en la zona plástica, obviamente más rígida. De continuar aumentando el valor de la fuerza aplicada y no haber una adecuada respuesta de estrés, se produce el fallo catastrófico de la deformación, lo que ocasiona la rotura del objeto en cuestión (1). 


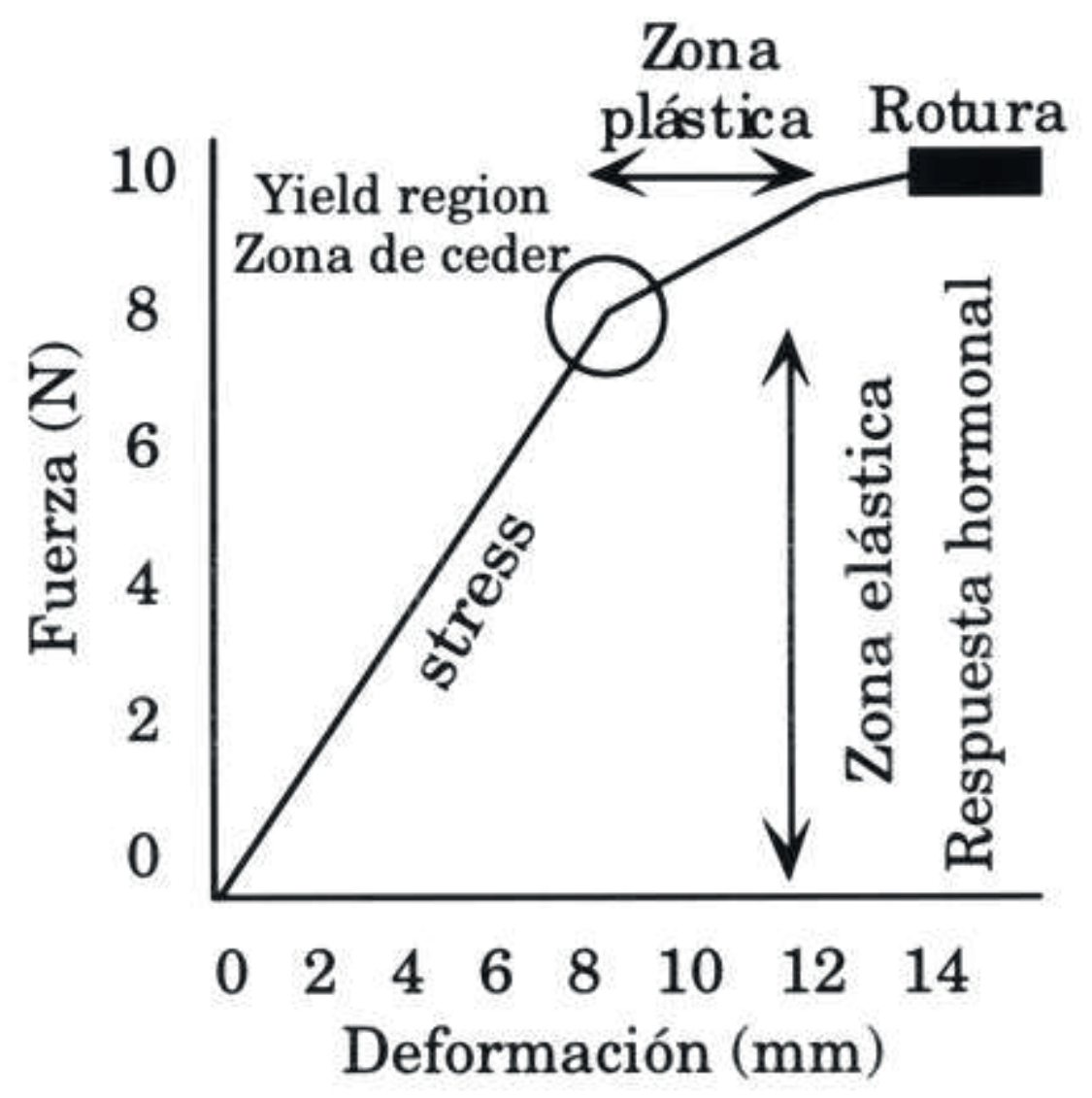

Figura 1. Relación entre la fuerza aplicada a un objeto y la deformidad que ocasiona (strain), dependientes del estrés o resistencia del objeto a la fuerza aplicada, que se caracteriza por una zona de "buena oposición" (zona elástica), una límite o de "fatiga" (yield region) y una zona plástica o de mínima adaptación/oposición que, de persistir, da lugar a la catastrófica rotura o "desequilibrio mental". Junto a la zona elástica, se incluye la respuesta hormonal fisiológica, que ocurre en todas las especies animales, ante la existencia de una "fuerza emocional".

En este principio físico y puramente biomecánico hay que distinguir varios conceptos:

- el tipo de fuerza aplicada: de tensión o estiramiento, de compresión o de torsión 
- y a su vez la característica de dicha fuerza: repentina (brusca) o continuada (paulatina), de forma lineal o progresiva

- y la intensidad de la fuerza: mínima, moderada o alta.

Frente a estas características de la fuerza aplicada, debemos tomar en consideración, las propias del objeto al que se aplica dicha fuerza, las que son estructurales y materiales; las materiales son las más específicas del objeto (acero, aluminio, caucho, corcho, etc.) y las estructurales, independientemente de las materiales, van a subordinarse en gran parte a la forma geométrica del objeto y a la denominada anisotropía (es obvio que no es lo mismo la resistencia de una bola de acero que la de un lingote, aún siendo los dos de acero y del mismo peso. En electrónica ocurre lo mismo, en un principio un circuito se opone al estímulo que quiere propagarse en su seno, para posteriormente ceder o, de ser muy intenso el estímulo, fundir o romper el circuito. Estas similitudes vienen a demostrarnos la importancia de unos simples principios físicos para comprender, de forma adecuada, unos mecanismos que parecen complejos en cuanto al funcionamiento ya sea mecánico o animal.

En medicina, relativo al estrés humano debemos considerar el strain, estrictamente hablando, en dos aspectos distintos: $1^{\circ}$. Como una reacción física a una determinada fuerza aplicada a nuestro organismo, en este caso es lo mismo que el mecánico, y $2^{\circ}$. Como el strain o la capacidad individual de deformación o adaptación al estrés, secundario a una fuerza o carga "emocional" aplicada a nuestra psiquis. Físicamente hablando, como ya hemos comentado, al igual que en biomecánica, esta capacidad es dependiente de la estructura y el material del que está compuesto el objeto. Podemos decir que, para comprender la similitud en el ejemplo con pocas diferencias, la estructura y material del cuerpo humano son superponibles en todos los individuos; la única diferencia es la capacidad psíquica, equivalente aquí al strain psíquico, de 
adaptarse al "estrés" como consecuencia de una determinada carga emocional, única circunstancia que en los objetos sin mente no está presente. Por ello es fácil deducir que en los humanos la capacidad de adaptación al estrés viene determinada, de forma prioritaria, por su equilibrio psíquico determinado por la "amplitud" de su strain o capacidad de adaptarse, $\mathrm{o}$

de dominio de la "deformación"; así la misma fuerza emocional puede generar un distinto estrés en individuos diferentes dependiendo de su capacidad psíquica de respuesta, es decir de su strain psíquico. La muerte de un familiar puede generar un dolor incontrolado en un miembro de la familia, mientras que en otros induce una simple resignación.

Esto nos lleva a concluir que el estrés humano es mayoritariamente dependiente, ante iguales fuerzas a las que se puede someter a un individuo, de su strain o capacidad psíquica; a mayor equilibrio es la misma mayor capacidad de respuesta para oponerse a la carga emocional y viceversa. Consecuentemente el estrés humano es principalmente una consecuencia psíquica del propio individuo y es obvio que el más débil psíquicamente hablando, sufrirá un mayor estrés ante la misma fuerza.

Otros factores son muy importantes al valorar el estrés en la especie humana, como es la capacidad de respuesta hormonal del organismo a la tensión emocional, lo que también podríamos denominar como el strain humoral. Ésta se realiza preferentemente a través de la elevación de las catecolaminas y los esteroides adrenales; en unos individuos puede ocasionar un total agotamiento de la capacidad de secreción, entrando en lo que se denomina "surmenage" o estado de agotamiento general con acusada adinamia y necesidad de descanso aislado y sin perturbaciones, otros pueden sentirse menos agotados, simplemente cansados, ligeramente adinámicos y otros mínimamente "nerviosos" o desconcertados sin saber definir de forma "adecuada" su estado anímico, pero que notan alterado. 
En definitiva, la deformidad orgánica (strain) que induce la carga emocional y a la que se opone el estrés del individuo, entrará en la zona plástica, dependiendo de una mayor o menor capacidad de "deformidad o adaptación" psíquica del individuo, que hace que unos, por ser menor dicho strain psíquico, se fracturen ocasionando alteraciones patológicas, mientras que en otros eso no llegue a ocurrir.

Como vemos una base física nos explica el condicionante psíquico del estrés y las amplias variaciones del mismo independientes de la fuerza o carga emocional que ocasionarán un mayor o menor strain (deformidad), dependiendo, "psíquicamente hablando", de la amplitud de adaptación mental del individuo, o strain psíquico.

\section{REFERENCIAS}

1. Bouxsein ML, Augat P. "Biomechanism of bone". En: Njeh CF, Hans D, Fuerst T, Glüer CC, Genant HK, eds. "Quantitative ultrasound". Martin Dunitz, London. 1999:21-46. 